(8) PRLPRT/10/93

\title{
Cosmic Ray and TeV Gamma Ray Generation by Quasar Remnants
}

\author{
Elihu Boldt* and Michael Loewenstein ${ }^{* \dagger}$ \\ * Laboratory for High Energy Astrophysics \\ NASA Goddard Space Flight Center \\ Greenbelt, $M D, U S A$ \\ †University of Maryland Department of Astronomy \\ College Park MD, USA
}

\begin{abstract}
Results from new broadband (radio to X-ray) high-resolution imaging studies of the dormant quasar remnant cores of nearby giant elliptical galaxies are now shown to permit the harboring of compact dynamos capable of generating the highest energy cosmic ray particles and associated curvature radiation of $\mathrm{TeV}$ photons. Confirmation would imply a global inflow of interstellar gas all the way to the accretion powered supermassive black hole at the center of the host galaxy.
\end{abstract}

\section{COMPACT DYNAMOS}

The quasar remnant cores of nearby giant elliptical galaxies NGC 4486 (M87), 1399, 4649 and 4472 are the sites of supermassive black holes $\left(>10^{9} \mathrm{M}_{\odot}\right)$. These MDOs (massive dark objects) could harbor compact dynamos capable of generating the highest energy cosmic rays [1]. For an accretion process involving an equipartition magnetic field near the event horizons of the underlying putative spun-up black holes, the proton energy achievable with such compact dynamos could well be $\geq 10^{20} \mathrm{eV}$, for situations where the principal loss mechanism is limited to curvature radiation.

\section{SETTING}

Recent estimates of the SED (spectral energy distribution) of ambient core photons lead to the conclusion that, except for M87, the energy losses arising from photo-pion production in proton collisions with these target photons are relatively small for the objects considered [2]. See Table 1 for a summary of the three (electromagnetically dark) galactic core sites of potential cosmic ray generation $\left(\geq 10^{20}\right.$ $\mathrm{eV}$ ) addressed here. All these correspond to an accretion rate of $\sim 1 \mathrm{M}_{\odot} \mathrm{yr}^{-1}$, comparable to the estimated Bondi rate and mass loss rate of giant ellipticals [3]. 
TABLE 1. Supermassive Black Hole Nuclei in Nearby Galaxies:

Potential Cosmic Ray / TeV Gamma Ray Generators

\begin{tabular}{lccc}
\hline Host Galaxy & NGC 1399 & NGC 4649 & NGC 4472 \\
\hline Host Cluster & Fornax & Virgo & Virgo \\
Distance (Mpc) & 29 & 18 & 18 \\
Black Hole Mass $\left(10^{9} \mathrm{M}_{\odot}\right)$ & 5.2 & 3.9 & 2.6 \\
Bondi Accretion $\left(\mathrm{M}_{\odot} \mathrm{yr}^{-1}\right)$ & 3.0 & 1.4 & 0.7 \\
Bulge Mass $\left(10^{12} \mathrm{M}_{\odot}\right)$ & 0.32 & 0.54 & 0.84 \\
LObserved $/ \mathrm{L}_{\mathrm{Bondi}}$ & $2 \times 10^{-6}$ & $3 \times 10^{-5}$ & $10^{-5}$ \\
Magnetic field (Tesla) & 0.44 & 0.40 & 0.43 \\
emf $\left(10^{20}\right.$ volts) & 21 & 14 & 10 \\
Proton Energy (ZeV) & 0.24 & 0.20 & 0.17 \\
Photon Energy (TeV) & 6.4 & 5.1 & 4.5 \\
\hline
\end{tabular}

Photon energy is the mean value for generated curvature radiation.

The black hole and galactic bulge masses are from [4]. The distances, Bondi accretion rate and $\mathrm{L}_{\text {Observed }} / \mathrm{L}_{\text {Bondi }}$ are from [5].

If these sites are found to be sources of such cosmic rays, it would imply the presence of a global inflow of interstellar gas that persists from large galactic radii all the way into the very center of the host galaxy. Thus while (hot) protogalactic gas may be the source for the initial rapid growth and quasar-epoch fueling of massive black holes [6], the stellar population of the galaxy at large may be responsible for 'feeding the monster' [7] during the present epoch; an era where the quieter power output may very well be characterized by ultra high energy particles and their associated $\mathrm{TeV}$ gamma radiation.

\section{GAMMA-RAY TRACER}

Substantial $\mathrm{TeV} \gamma$-ray curvature radiation is a necessary consequence of cosmic ray acceleration by such black hole dynamos $[8,9]$. The luminosity in this gammaradiation is more than that represented by the energetic cosmic rays generated. This means that these sources could be located with the precision of photon astronomy; in comparison, locating them by charged particle arrival direction would be severely limited by intervening magnetic field deflections in the IGM $[10,11]$. Depending on the actual number of MDOs involved, those dynamos in the likely subset $\left(>10^{9} \mathrm{M}_{\odot}\right)$ responsible for the observed ultra-high energy cosmic ray flux $\left(\geq 10^{20} \mathrm{eV}\right)$ could have an average luminosity up to $\sim 10^{43} \mathrm{erg} \mathrm{s}^{-1}$ in associated $\mathrm{TeV}$ curvature radiation, exceeding the total luminosity in lower energy photons. Searching the apparently dormant cores of nearby giant elliptical galaxies for evidence of $\mathrm{TeV} \gamma$-rays characteristic of ultra-relativistic particle acceleration could signal the beginning of a new avenue for gamma-ray astronomy. 


\section{REFERENCES}

1. Boldt, E., and Ghosh, P., MNRAS 307, 491 (1999).

2. Boldt, E., and Loewenstein, M., MNRAS 316, L29 (2000).

3. Mathews, W. G., $A J$ 97, 42 (1989).

4. Magorrian, J. et al., $A J$ 115, 2285 (1998).

5. Di Matteo, T., Quataert, E., Allen, S. W., Narayan, R., and Fabian, A. C. MNRAS 311, 507 (2000).

6. Nulsen, P. E. J.. and Fabian, A. C., MNRAS 311, 346 (2000).

7. Gunn, J., Active Galactic Nuclei, edited by C. Hazard and S. Mitton, Cambridge University Press, Cambridge, 1979, p. 213.

8. Levinson, A., Phys. Rev. Lett. 85, 912 (2000).

9. Levinson, A., this meeting.

10. Farrar, G. and Piran, T., Phys. Rev. Lett. 84, 3527 (2000).

11. Kronberg, P., this meeting. 
